\title{
New Detector Architecture for Electron Microscopes with SDDs
}

\author{
H.Soltau, ${ }^{*}$ O.Jaratschin, ${ }^{* *}$ A.Liebl,** A.Niculae, ${ }^{*}$ A.Simsek, ${ }^{* *}$ \\ R.Eckhard, ${ }^{*}$ K.Hermenau, ${ }^{*}$ P.Lechner, ${ }^{*}$ G.Lutz, ${ }^{*}$ G.Schaller, ${ }^{* * *}$ F.Schopper, ${ }^{* * *}$ L.Strüder***
}

*PNSensor GmbH, Römerstr. 28, D-80803 München, Germany

**PNDetector GmbH, Emil-Nolde-Str.10, D-81735 München, Germany

***MPI Halbleiterlabor, Otto-Hahn-Ring 6, D-81739 München, Germany

Silicon Drift Detectors (SDDs) are used as X-ray sensors in Scanning and Transmission Electron Microscopes. They provide excellent performance with regard to energy resolution, light element performance, peak-to-background ratio and count rate capability. Within the last two years SDDs have substituted $\mathrm{Si}(\mathrm{Li})$ Detectors for all new EDX systems for the two facts of higher measurement speed and the avoidance of liquid nitrogen cooling.

The Semiconductor Laboratory of the Max-Planck-Institutes of Physics and Extraterrestrial Physics (MPI/HLL) and the company PNSensor are designing and fabricating SDDs with integrated FET for this purpose since many years [1]. Because the sensors are fabricated monolithically in a planar silicon process (see Fig.1a) and because they provide very good energy resolution at high temperatures thanks to the ultrapure process and the integration of the FET (see Fig.1b) they allow a whole variety of new possibilities of design and architecture for X-ray and electron analysis tools, adapted to the specific needs and the geometric boundary conditions especially in electron microscopes.

As an example Fig.2a shows a SDD with an area of $60 \mathrm{~mm}^{2}$ to be placed directly below the pole shoe of a SEM to detect X-rays with high quantum efficiency. The single cells of the 4-element device adapt in their kidney shape to the round hole of the pole shoe. This detector can be arranged directly at the pole shoe or in a small distance stand-alone and rectractable. Fig.2b shows a different shape of a $95 \mathrm{~mm}^{2}$ double cell SDD also well fitting to the pole shoe opening. Besides the design of the sensors themselves the SDD mountage is very flexible and accounts for the small amount of space available in an electron microscope. The fact that for SDDs with integrated FET an external bulky cooling mechanism is not necessary to reach optimum performance becomes very important. As demonstrated in Figs.3a and $\mathrm{b}$ the detector can be built up in a very slim version connected electrically by a small flexlead and cooled by thermal vias and a peltier element at one side only. This detector architecture allows various integration possibilities into electron microscopes adapted to the specific needs especially if regarded that there are almost no limits to the design of the sensor and that combination with an ASIC is possible to reach an even higher level of integration.

A new SDD architecture of that kind can be used for electrons as well as X-rays thereby measuring both, medium and low energy X-rays, by the use of hard- and software filters [2]. Fig.4a shows an electron spectrum in comparison with an X-ray spectrum of that same energy. Measurements of back scattered electrons and X-ray fluorescence radiation could also be combined in one detector using different cells for electrons and X-rays in circulars of growing radii as visualized in Fig. 4b.

[1] H.Soltau et al, Microscopy and Microanalysis 14, 1156 (2008).

[2] L. Strüder et al, Microscopy and Microanalysis 14, 76 (2008).

[3] T. Armbrust, FZ Karlsruhe, Master Thesis, IKP, 2004. 

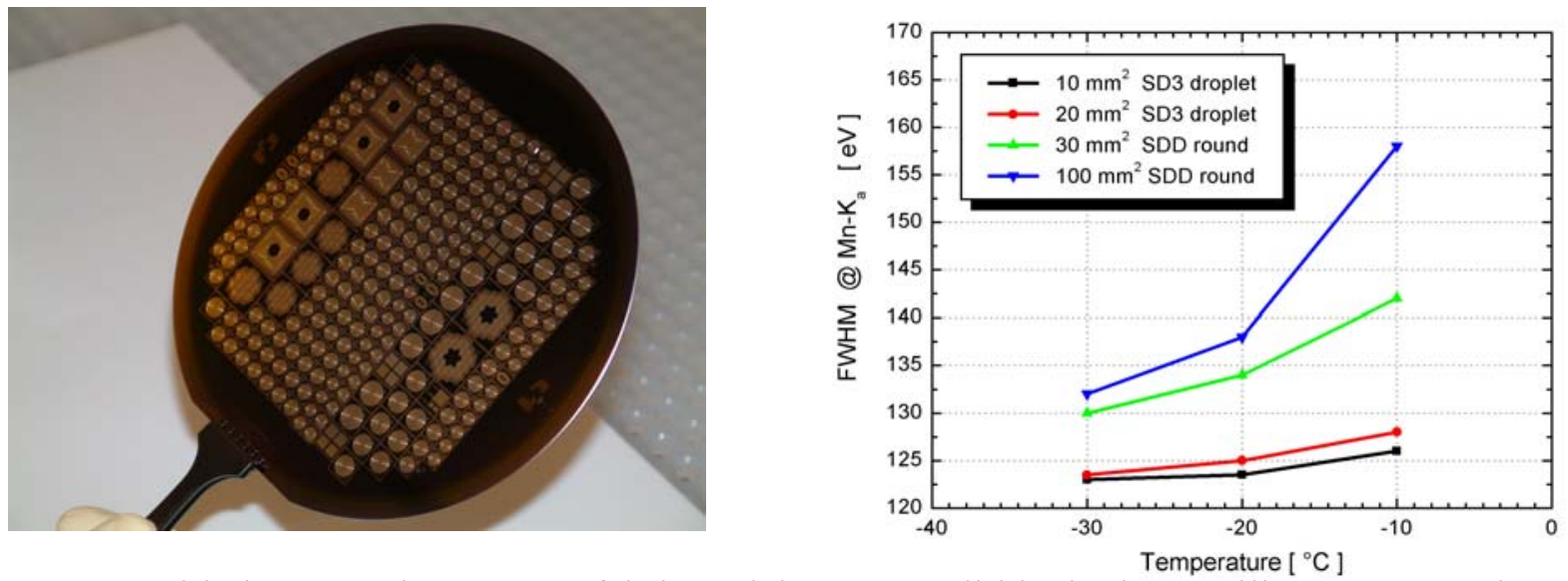

FIG.1. SDDs with integrated FET are fabricated in a monolithical planar silicon process in an ultrapure technology providing excellent energy resolution at high temperatures.
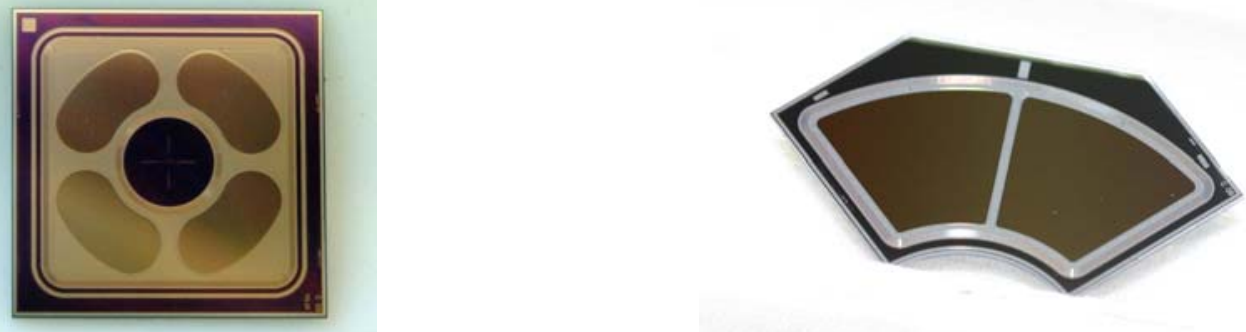

FIG.2. Planar technology allows almost unlimited varieties of sensor designs for X-ray and electon analysis tools adapted to the specific needs and the boundary conditions of electron microscopes.

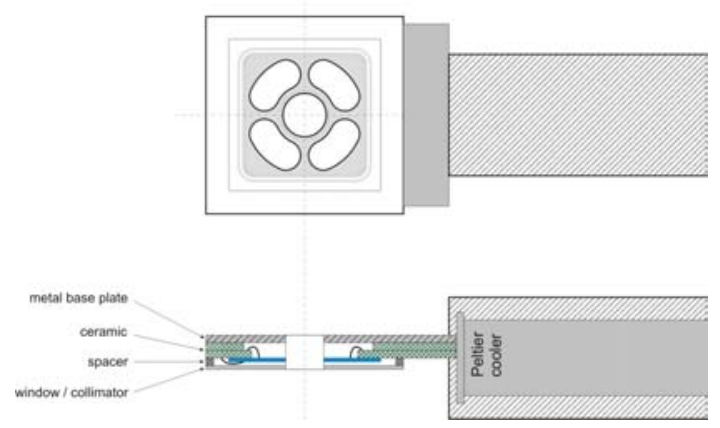

FIG.3. The SDD mountage is very flexible and accounts for the small amount of space available in an electron microscope.

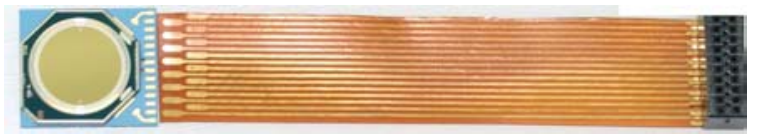

FIG.4. SDDs can be used to measure both, electrons and X-rays [3]. The sensor layout can foresee different cells for acquisiton or identical ones, using soft- and hardware filters.
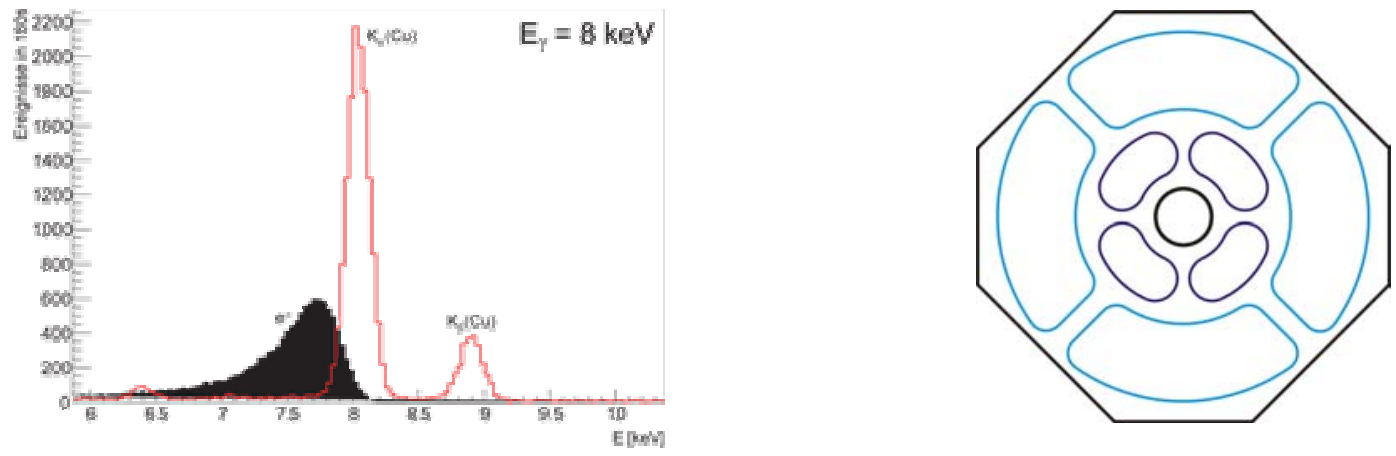